品 
Trends in Mathematics is a series devoted to the publication of volumes arising from conferences and lecture series focusing on a particular topic from any area of mathematics. Its aim is to make current developments available to the community as rapidly as possible without compromise to quality and to archive these for reference.

Proposals for volumes can be sent to the Mathematics Editor at either

Birkhäuser Verlag

P.O. Box 133

$\mathrm{CH}-4010$ Basel

Switzerland

or

Birkhäuser Boston Inc.

675 Massachusetts Avenue

Cambridge, MA 02139

USA

Material submitted for publication must be screened and prepared as follows:

All contributions should undergo a reviewing process similar to that carried out by journals and be checked for correct use of language which, as a rule, is English. Articles without proofs, or which do not contain any significantly new results, should be rejected. High quality survey papers, however, are welcome.

We expect the organizers to deliver manuscripts in a form that is essentially ready for direct reproduction. Any version of TeX is acceptable, but the entire collection of files must be in one particular dialect of TeX and unified according to simple instructions available from Birkhäuser.

Furthermore, in order to guarantee the timely appearance of the proceedings it is essential that the final version of the entire material be submitted no later than one year after the conference. The total number of pages should not exceed 350. The first-mentioned author of each article will receive 25 free offprints. To the participants of the congress the book will be offered at a special rate. 


\section{Differential Equations with \\ Symbolic Computation}

Dongming Wang

Zhiming Zheng

Editors 
Editors:

Dongming Wang

School of Science

Beihang University

37 Xueyuan Road

Beijing 100083

China

and

Laboratoire d'Informatique de Paris 6

Université Pierre et Marie Curie - CNRS

8 , rue due Capitaine Scott

75015 Paris

France

e-mail: Dongming.Wang@lip6.fr
Zhiming Zheng

School of Science

Beihang University

37 Xueyuan Road

Beijing 100083

China

e-mail: zzheng@pku.edu.cn

2000 Mathematical Subject Classification 34-06; 35-06; 68W30

A CIP catalogue record for this book is available from the

Library of Congress, Washington D.C., USA

Bibliographic information published by Die Deutsche Bibliothek

Die Deutsche Bibliothek lists this publication in the Deutsche Nationalbibliografie; detailed bibliographic data is available in the Internet at http://dnb.ddb.de

ISBN 3-7643-7368-7 Birkhäuser Verlag, Basel - Boston - Berlin

This work is subject to copyright. All rights are reserved, whether the whole or part of the material is concerned, specifically the rights of translation, reprinting, re-use of illustrations, recitation, broadcasting, reproduction on microfilms or in other ways, and storage in data banks. For any kind of use permission of the copyright owner must be obtained.

(c) 2005 Birkhäuser Verlag, P.O. Box 133, CH-4010 Basel, Switzerland

Part of Springer Science+Business Media

Printed on acid-free paper produced from chlorine-free pulp. TCF $\infty$

Printed in Germany

ISBN-10: 3-7643-7368-7

e-ISBN: 3-7643-7429-2

ISBN-13: 976-3-7643-7368-9 


\section{Preface}

This book provides a picture of what can be done in differential equations with advanced methods and software tools of symbolic computation. It focuses on the symbolic-computational aspect of three kinds of fundamental problems in differential equations: transforming the equations, solving the equations, and studying the structure and properties of their solutions. Modern research on these problems using symbolic computation, or more restrictively using computer algebra, has become increasingly active since the early 1980s when effective algorithms for symbolic solution of differential equations were proposed, and so were computer algebra systems successfully applied to perturbation, bifurcation, and other problems. Historically, symbolic integration, the simplest case of solving ordinary differential equations, was already the target of the first computer algebra package SAINT in the early 1960s.

With 20 chapters, the book is structured into three parts with both tutorial surveys and original research contributions: the first part is devoted to the qualitative study of differential systems with symbolic computation, including stability analysis, establishment of center conditions, and bifurcation of limit cycles, which are closely related to Hilbert's sixteenth problem. The second part is concerned with symbolic solutions of ordinary and partial differential equations, for which normal form methods, reduction and factorization techniques, and the computation of conservation laws are introduced and used to aid the search. The last part is concentrated on the transformation of differential equations into such forms that are better suited for further study and application. It includes symbolic elimination and triangular decomposition for systems of ordinary and partial differential polynomials. A 1991 paper by Wen-tsün Wu on the construction of Gröbner bases based on Riquier-Janet's theory, published in China and not widely available to the western readers, is reprinted as the last chapter. This book should reflect the current state of the art of research and development in differential equations with symbolic computation and is worth reading for researchers and students working on this interdisciplinary subject of mathematics and computational science. It may also serve as a reference for everyone interested in differential equations, symbolic computation, and their interaction.

The idea of compiling this volume grew out of the Seminar on Differential Equations with Symbolic Computation (DESC 2004), which was held in Beijing, China in April 2004 (see http://www-calfor.lip6.fr/ ${ }^{\sim}$ wang/DESC2004) to facilitate the interaction between the two disciplines. The seminar brought together active researchers and graduate students from both disciplines to present their work and to report on their new results and findings. It also provided a forum for over 50 participants to exchange ideas and views and to discuss future development and cooperation. Four invited talks were given by Michael Singer, Lan Wen, Wen-tsün $\mathrm{Wu}$, and Zhifen Zhang. The enthusiastic support of the seminar speakers and the 
high quality of their presentations are some of the primary motivations for our endeavor to prepare a coherent and comprehensive volume with most recent advances on the subject for publication. In addition to the seminar speakers, several distinguished researchers who were invited to attend the seminar but could not make their trip have also contributed to the present book. Their contributions have helped enrich the contents of the book and make the book beyond a proceedings volume. All the papers accepted for publication in the book underwent a formal review-revision process.

DESC 2004 is the second in a series of seminars, organized in China, on various subjects interacted with symbolic computation. The first seminar, held in Hefei from April 24-26, 2002, was focused on geometric computation and a book on the same subject has been published by World Scientific. The third seminar planned for April 2006 will be on symbolic computation in education.

The editors gratefully acknowledge the support provided by the Schools of Science and Advanced Engineering at Beihang University and the Key Laboratory of Mathematics, Informatics and Behavioral Semantics of the Chinese Ministry of Education for DESC 2004 and the preparation of this book. Our sincere thanks go to the authors for their contributions and cooperation, to the referees for their expertise and timely help, and to all colleagues and students who helped for the organization of DESC 2004.

Beijing

Dongming Wang

May 2005

Zhiming Zheng 


\section{Contents}

Symbolic Computation of Lyapunov Quantities and the Second Part

of Hilbert's Sixteenth Problem ...................................... 1

Stephen Lynch

Estimating Limit Cycle Bifurcations from Centers $\ldots \ldots \ldots \ldots \ldots \ldots \ldots \ldots 23$

Colin Christopher

Conditions of Infinity to be an Isochronous Center for a Class of

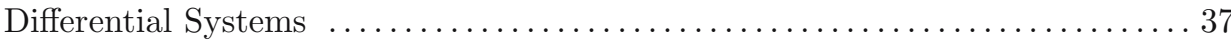

Wentao Huang and Yirong Liu

Darboux Integrability and Limit Cycles for a Class of Polynomial

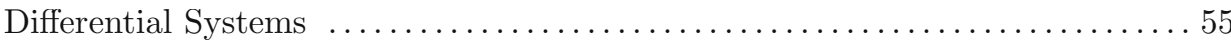

Jaume Giné and Jaume Llibre

Time-Reversibility in Two-Dimensional Polynomial Systems $\ldots \ldots \ldots \ldots \ldots 67$

Valery G. Romanovski and Douglas S. Shafer

On Symbolic Computation of the LCE of $\mathbf{N}$-Dimensional Dynamical

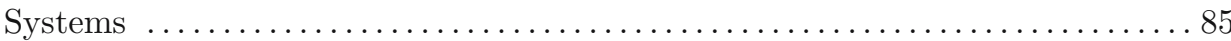

Shucheng Ning and Zhiming Zheng

Symbolic Computation for Equilibria of Two Dynamic Models ............ 109

Weinian Zhang and Rui Yan

Attractive Regions in Power Systems by Singular Perturbation Analysis

Zhujun Jing, Ruiqi Wang, Luonan Chen and Jin Deng

Algebraic Multiplicity and the Poincaré Problem

Jinzhi Lei and Lijun Yang

Formalizing a Reasoning Strategy in Symbolic Approach to Differential

Equations

Shilong $\mathrm{Ma}$

Looking for Periodic Solutions of ODE Systems by the Normal Form

Method

Victor F. Edneral 
viii

Algorithmic Reduction and Rational General Solutions of First Order


Guoting Chen and Yujie Ma

Factoring Partial Differential Systems in Positive Characteristic . . . . . . . . . 213

Moulay A. Barkatou, Thomas Cluzeau and Jacques-Arthur Weil

On the Factorization of Differential Modules

Min $W u$

Continuous and Discrete Homotopy Operators and the Computation of Conservation Laws

Willy Hereman, Michael Colagrosso, Ryan Sayers, Adam Ringler,

Bernard Deconinck, Michael Nivala and Mark Hickman

Partial and Complete Linearization of PDEs Based on Conservation Laws

Thomas Wolf

CONSLAW: A Maple Package to Construct the Conservation Laws for Nonlinear Evolution Equations

Ruo-Xia Yao and Zhi-Bin Li

Generalized Differential Resultant Systems of Algebraic ODEs and

Differential Elimination Theory

Giuseppa Carrá Ferro

On "Good" Bases of Algebraico-Differential Ideals Wen-tsün $W u$

On the Construction of Groebner Basis of a Polynomial Ideal Based on Riquier-Janet Theory

Wen-tsün $W u$

Index 